\title{
MAPPING SOUTH BALTIC NEAR-SHORE BATHYMETRY USING SENTINEL-2 OBSERVATIONS
}

\author{
Andrzej Chybicki \\ Gdansk University of Technology, Poland
}

\begin{abstract}
One of the most promising new applications of remote observation satellite systems $(R O)$ is the near-shore bathymetry estimation based on spaceborn multispectral imageries. In recent years, many experiments aiming to estimate bathymetry in optically shallow water with the use of remote optical observations have been presented. In this paper, optimal models of satellite derived bathymetry (SDB) for relatively turbid waters of the South Baltic Sea were presented. The obtained results were analysed in terms of depth error estimation, spatial distribution, and overall quality. The models were calibrated based on sounding (in-situ) data obtained by a single-beam echo sounder, which was retrieved from the Maritime Office in Gdynia, Poland. The remote observations for this study were delivered by the recently deployed European Space Agency Sentinel-2 satellite observation system. A detailed analysis of the obtained results has shown that the tested methods can be successfully applied for the South Baltic region at depths of 12-18 meters. However, significant limitations were observed. The performed experiments have revealed that the error of model calibration, expressed in meters (RMSE), equals up to 10-20\% of the real depth and is, generally, case dependent. To overcome this drawback, a novel indicator of determining the maximal SDB depth was proposed. What is important, the proposed SDB quality indicator is derived only on the basis of remotely registered data and therefore can be applied operationally.
\end{abstract}

Keywords: near-shorel satellite, derived, bathymetry, Sentinel, multispectra, observation

\section{INTRODUCTION}

Satellite remote multispectral systems provide valuable large- and local-scale observations of optical and thermal properties of Earth's surface. One of the most promising new applications of the remote observation satellite systems (RO) are near-shore bathymetry estimations. RO observations are an interesting approach because they can provide relatively low-cost information on shallow water bathymetry, compared to other known bathymetry retrieval techniques, such as Lidar scanning (LS), multibeam systems (MBS) and single-beam echo sounder (SBE). The above methods, particularly LS and MBS, provide high resolution and accurate data, but surveying in those cases is usually expensive and time consuming [1] [2]. With the development of optical and thermal satellite sensors for land and sea observation imagers, new applications of RO arise.

In recent years many experiments aiming to estimate bathymetry in optically shallow waters with the use of remote optical observation have been presented. Basically, two fundamental models of determining the bathymetry from optical imagery are defined, namely: the empirical optical band ratio transform algorithm proposed by Stumpf [3] and a more analytical approach proposed by Lyzenga $[4,5]$ and Philpot [6]. Both of these models assume that the radiation in optical bands is absorbed by water and reflected from the bottom. However, the scale of this process differs depending on the wavelength. Therefore, the ratio of the observed radiances of at least two optical bands is to be used to retrieve the information about the bottom depth.

During this process, many factors constitute limitations of these methodologies. When the bottom reflectance and light attenuation of water is stable over the analysed area, depth estimates can be relatively easily made by modelling the depth of light penetration based on the amount of reflectance measured by the satellite. Having known the multiple visiblewavelength spectral bands, the effects of seafloor reflectance variability and water turbidity can be reduced. However, water turbidity is still one of the most important factors in the process of obtaining satellite derived bathymetry (SDB).

Therefore, most of the experiments made in this area have focused on testing fields and datasets that satisfy the 
abovementioned criteria. For instance, Sandidge and Holyer used the Airborn Visible/Infrared scanner to derive the bathymetry for waters of Florida, USA [7]. The observations of optically shallow waters near the Bahama islands were also analysed by Adler-Godlen [8], Sheng Ma [9], and others i.e. [10][11][12][13]. This paper presents optimal models of satellite based bathymetry derivation developed for relatively turbid waters of the South Baltic Sea. The research involved Sentinel-2 data, as well as log-ratio and analytical approaches making use of inverse transform optimization methods. The results obtained using these two models were then compared in terms of depth error estimation, spatial distribution, and quality. The model was calibrated on the basis of the sounding (in-situ) data obtained by a single-beam echo sounder. The calibration data was retrieved from the Maritime Office in Gdynia, Poland, which is the local entity of official Marine Administration in Poland.

\section{METHODS}

Bathymetry estimation from satellite observations involves extracting the bottom radiance from the measured waterleaving reflectance. The reflectance $\mathrm{R}$ is defined as the ratio of the radiance leaving the water surface to the downwelling irradiance just above the water surface. It is a feature that describes light absorption related optical properties of the surface, as well as scattering properties of the constituents in the water, and bottom albedo and depth. The fundamental physical principle in the process of deriving bathymetry using satellite observations bases on the phenomena of light pass attenuation in the water column, and bottom reflection and scattering (Fig. 1).

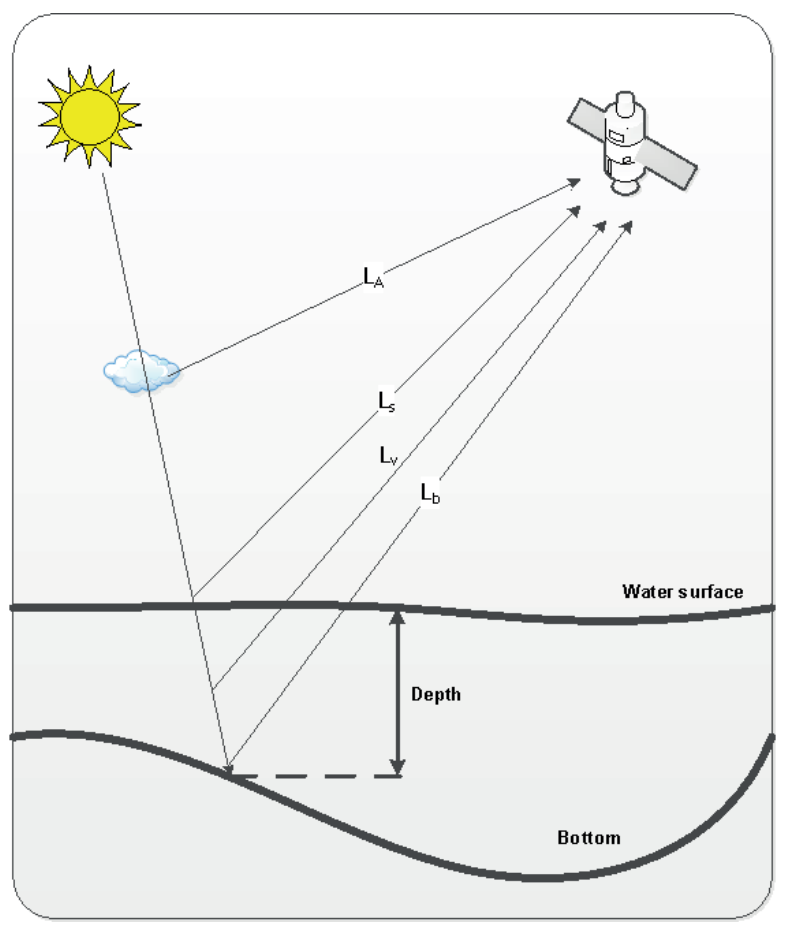

Fig. 1. Physical principle of SDB model
As shown in Fig. 1, this principle can be divided into four basic components of the energy registered at the satellite sensor [14] which are: the bottom radiance $L_{B}$, the subsurface volumetric radiance $L_{v}$, the specular radiance $L_{s}$, and the atmospheric path radiance $L_{A}$. This can be written down as:

$$
L_{T O A}=L_{B}+L_{V}+L_{S}+L_{A}
$$

where the radiance $\mathrm{L}_{\mathrm{TOA}}$ registered at the sensor includes the atmospheric scattering $L_{A}$ and the subsurface volumetric radiance $L_{v}$ resulting from volume scattering in water and its organic/inorganic constituents (e.g. sediment and chlorophyll). The surface radiance $L_{S}$ is caused by the reflection of optical energy from the water surface, including possible sunglint effects. Finally, $L_{B}$ is the result of energy reflection from the seabed, which holds the information about bottom scattering characteristics and water depth. In this context, in order to derive the bottom depth from satellite observation, disaggregating the bottom and volumetric radiance from the total radiance is crucial.

Basically, there are two fundamental models for obtaining SDB. Both of them apply mechanisms to remove $L_{p}$ and specular effects, and minimize the variability of volumetric scattering effects [15][16]. In most approaches this is achieved by assuming that bottom radiance in fully deep water equals zero. Then, the total radiance (or reflectance) over opticallydeep water $\left(L_{\infty}\right.$ or $\left.R_{\infty}\right)$, represents the combined effect of subsurface volumetric radiance, specular radiance, and atmospheric path radiance. After atmospheric and sunglint corrections, the deep-water radiance contains only subsurface volumetric radiance. Assuming that the subsurface volumetric radiance in shallow water and atmospheric absorption is the same as that in the adjacent deep water, the optically deepwater radiance recorded by the remote sensor can be used to correct the subsurface volumetric radiance in shallow water.

In order to minimize depth estimation errors, a possibly largest number of wavelength bands with smallest attenuation should be used. The maximal derivation depth is limited by water turbidity (caused by suspended sediments, chlorophyll, and organic particles) and the wavelength registered by the sensor. Therefore, the basic band used for SBD is blue light spectrum (440 to $540 \mathrm{~nm}$ ) as it has the smallest attenuation and can penetrate water up to $30 \mathrm{~m}$ in optimal conditions. Longer wavelengths (green and red) attenuate rapidly in water, as a consequence of which green light (500-600 nm) can penetrate to a maximum depth of approximately $15 \mathrm{~m}$, red light $(600-700 \mathrm{~nm})$ to $5 \mathrm{~m}$, and near infrared $(700-800$ $\mathrm{nm})$ to as little as $0.5 \mathrm{~m}$ [17].

\section{OPTICAL BAND RATIO BATHYMETRY RETRIEVAL MODEL}

The first of the above described approaches [3], based on a log-ratio equation, is described by the following equation: 


$$
z_{\text {est }}=m_{1} \frac{\ln \left(R\left(\chi_{i}\right)\right)}{\ln \left(R\left(\chi_{j}\right)\right)}+m_{0}
$$

where $z_{\text {est }}$ is the satellite derived bathymetry depth, $m_{0}$ and $m_{1}$ are the coefficients of the model, and $R\left(\lambda_{i}\right)$ and $R\left(\lambda_{j}\right)$ are the remote sensing radiances for optical bands $\lambda_{i}$ and $\lambda_{j}$. In this model, the bottom depth is estimated on the basis of light attenuation phenomena, as the attenuation of the incoming shortwave radiation varies spectrally. This effect can be observed in spectral bands.

\section{ANALYTICAL INVERSION MODEL}

The local inversion model is derived directly from the simplified radiation equation for optically shallow waters (3):

$$
L_{T O A}=L_{\infty}\left[1-e^{-k z}\right]+A_{d} e^{-k z}+L_{A}+L_{S}
$$

where $A_{d}$ is the upwelling spectral radiance directly reflected from the bottom (before interacting with the overlaying water column), $k$ is the two-way attenuation coefficient, and $z$ is the depth. In this context, the expression $A_{d} e^{-k z}$ represents the energy attenuation effect resulting from energy passing through the water column of known depth $z$. Assuming that the ratio of bottom reflectance between two spectral bands is constant for all bottom types within a given scene and the light attenuation variability caused by atmospheric effects is negligible for a given area, the depth estimated with the use of the following model can be expressed as:

$$
\mathrm{z}_{\mathrm{est}}=\alpha_{0}+\sum_{i=1}^{N} \alpha_{i} \ln \left[L\left(\lambda_{i}\right)-L_{\infty}\left(\lambda_{i}\right)\right]
$$

where $N$ is the number of spectral bands, $\alpha_{i}(i=1,2, \ldots N)$ are the constant coefficients derived during model calibration, and $L\left(\lambda_{i}\right)$ is the remote sensing radiance after atmospheric and sunglint corrections for spectral band $\lambda_{i}$. The use of natural logarithm in the expression makes the transformation linear to water depth and deepwater-corrected radiances of spectral bands.

\section{MATERIALS}

In this section, the description of input data for the algorithms used in the paper is outlined. The proposed algorithms utilize two types of input datasets, which are: multispectral imageries obtained from the Sentinel-2 satellite system that SDB is derived from, and the calibration dataset constructed from SBE surveys.

\section{SENTINEL-2 DATA}

Sentinel-2 (S2) is a two polar-orbiting satellite system that is the continuation of the SPOT and Landsat series of multispectral missions. Its main objective is to deliver highresolution optical and thermal operational observations for land/sea monitoring, emergency response, and security services [18]. Sentinel-2 is part of the European Space Agency (ESA) Copernicus programme and its data is provided via dedicated data dissemination frameworks, such as SciHub [19] or national Copernicus mirror sites [20][21].

Sentinel-2 provides systematic coverage of the globe between $56^{\circ} \mathrm{S}$ to $84^{\circ} \mathrm{N}$, with relatively high revisit frequency (every five days at the equator under the same viewing conditions). The spatial resolution for optical and NIR $(865 \pm 10 \mathrm{~nm})$ bands equals $10 \mathrm{~m} \times 10 \mathrm{~m}$ per pixel. In the case of the analysed area, data is delivered via the UTM $34 \mathrm{~N}$ projection grid. Sentinel-2 also delivers six NIR and SWIR bands with $20 \mathrm{~m} \times 20 \mathrm{~m}$ spatial resolution and three $60 \mathrm{~m}$ resolution bands in optical, NIR and SWIR ranges (Fig. 2).

The observation data from S2 is delivered by the mission Ground Segment, which provides processing schemes in four levels:

- Level-0 (L0) - raw compressed geometrically registered data

- Level-1 (L1) - divided into A, B, C and C sub-stages. The stage L1C provides geocoded uncompressed TOA reflectance after radiometric calibration, data correction, and geometric refinement.

- Level-2 (L2) - the stage can be performed with the use of dedicated processing software ([22][23]) and provides the bottom of the atmosphere reflectance.

\section{SWIR}

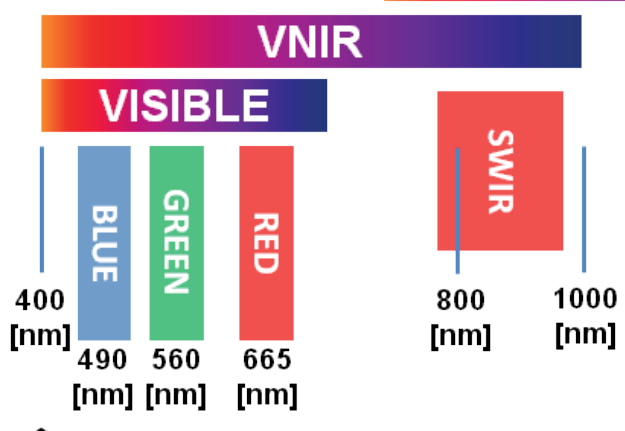

Wavelength [nm]

Fig. 2. Sentinel-2 MSI optical band characteristics.

\section{SOUNDING DATA}

The data for SDB model calibration was delivered by the Maritime Office in Gdynia (MAG), which is part of national maritime authority in Poland. Its duties cover such activities as: ensuring and monitoring maritime safety and security in the scope of inspections carried out by Flag State Control and Port State Control, monitoring of ships' traffic, sea routes and security of ship and port facilities, monitoring of ships' routes and waterways, management of waters, maritime spatial planning, and others. 
In addition to the above stated duties, MAG performs systematic Polish coast bathymetry surveys with the aid of different survey techniques, including SBE, MBS, and Lidar scanning. The data for the presented research was retrieved from near-shore SBE surveys made in 2011. The testing site covers $12 \mathrm{~km}$ of the South Baltic coast (Fig. 3).

Each survey is based on acoustic sounding profile depth measures, where each sounding within the profile is spaced by $10-20 \mathrm{~m}$. Each profile is perpendicular to the coast and starts 1800-2000m before the coastline, which corresponds to the bottom depth of about $15-20 \mathrm{~m}$. The profiles are parallel to each other and spaced by $500 \mathrm{~m}$ along the coast.
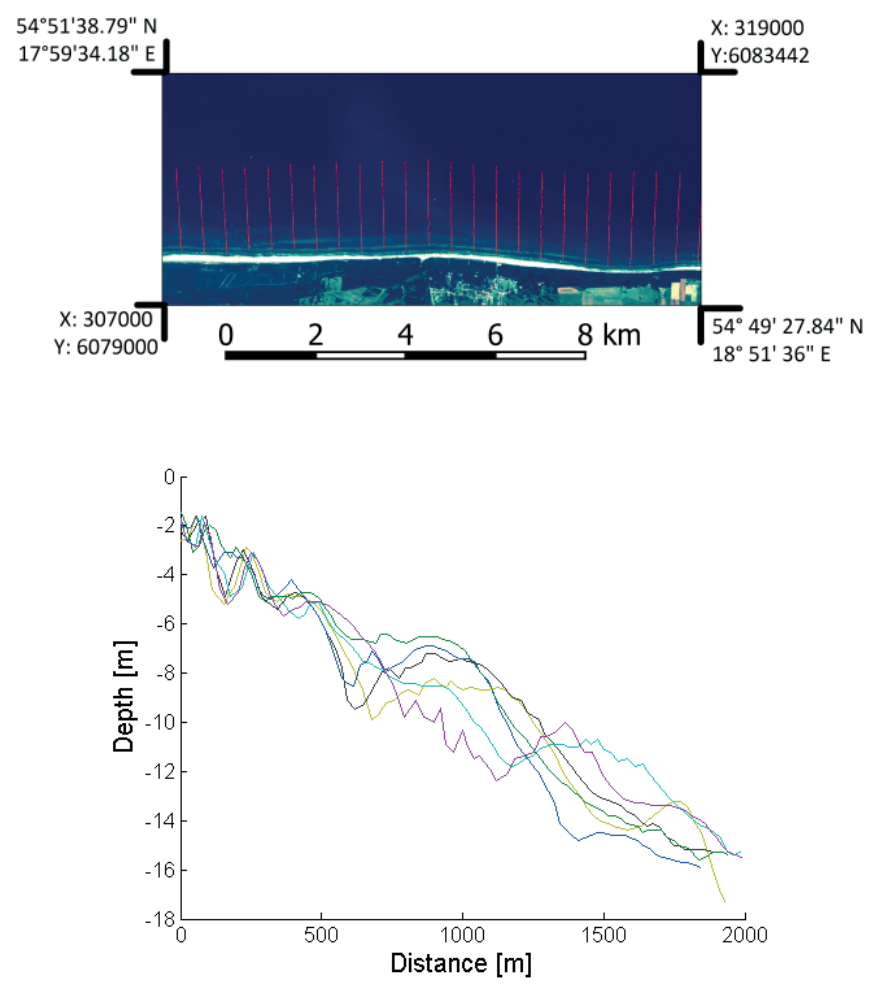

Fig. 3. Upper picture: calibration test site geographically positioned on the basis of composite RGB Sentinel-2 imagery in UTM 34N projection. Red dots represent SBE soundings profiles. Lower picture: plots of selected sounding profiles (the same as in the upper figure) as functions of distance from the shore.

\section{RESULTS}

In order to calibrate the proposed models of bathymetry retrieval, the sounding data described in the previous section was used. This process was based on visual and analytical inspection of the calibrating dataset. As it can be observed, the sounding in-situ observations contain not only underwater soundings but also some small number of in-situ measurements along the coast (above the water surface). Because of this, the in-situ observations with depth less than $0.5 \mathrm{~m}$ were removed from further analysis. The remaining data was compared to the remote Sentinel-2 observations acquired on $4^{\text {th }}$ March 2016, $9^{\text {th }}$ March 2016, 27 ${ }^{\text {th }}$ March 2016, and $6^{\text {th }}$ May 2016 under clean-sky conditions.

\section{LOG-RATIO MODEL CALIBRATION}

During log-ratio model calibration, for each sounding point the <observation, model value $>$ pair is built. Then, for each pair the model value is calculated using eq. (2) with initial values of $m_{0}=1$ and $m_{1}=0$. In the next step, this set of pairs is put under second degree polynomial regression in order to obtain optimal $m_{0}$ and $m_{1}$ values. The root mean square error of calibration and correlation is calculated as the quality indicator. The total number of calibration points equals 2074 (Fig. 4). Figures 4-7 show scatter plots of calibrated SDB corresponding to sounding data for different S2 observations.

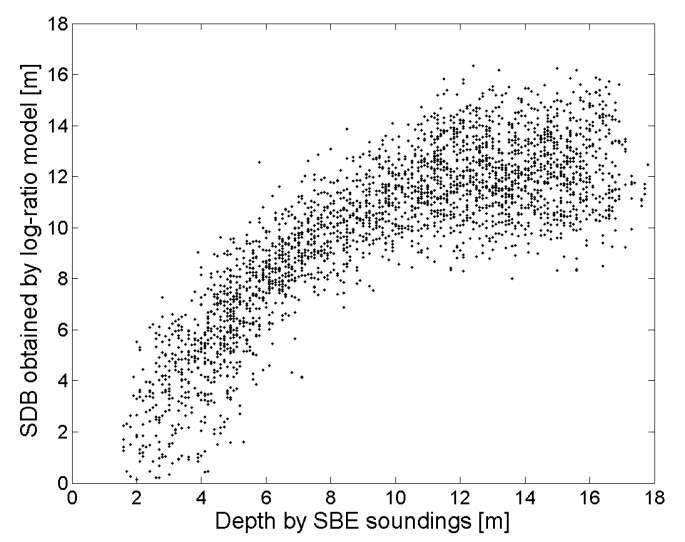

Fig. 4. Scatter plot of results obtained by optimized log-ratio model vs. depths obtained by SBE. Root mean square error was $R M S E=2.4231[\mathrm{~m}]$ and Pearson correlation coefficient was $R=0.8254$ Model was calibrated on the basis of satellite acquisition made on $4^{\text {th }}$ March 2016.

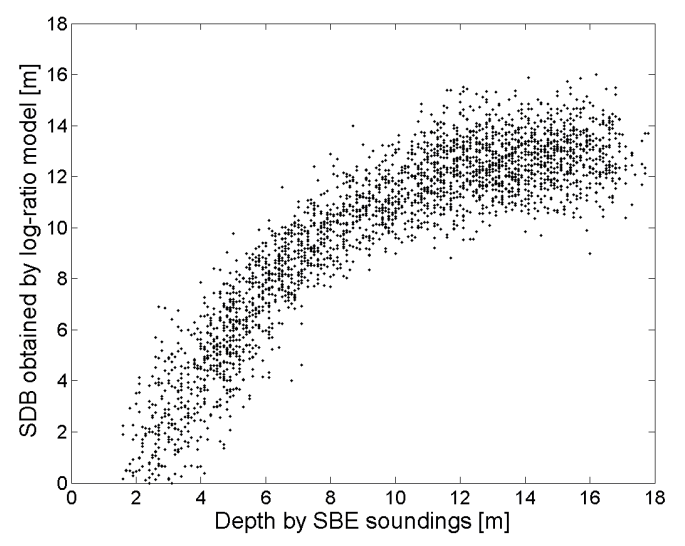

Fig. 5. Scatter plot of results obtained by optimized log-ratio model vs. depths obtained by SBE. Root mean square error was RMSE $=2.0555[\mathrm{~m}]$ and Pearson correlation coefficient was $R=0.8779$. Model was calibrated on the basis of satellite acquisition made on $9^{\text {th }}$ March 2016. 


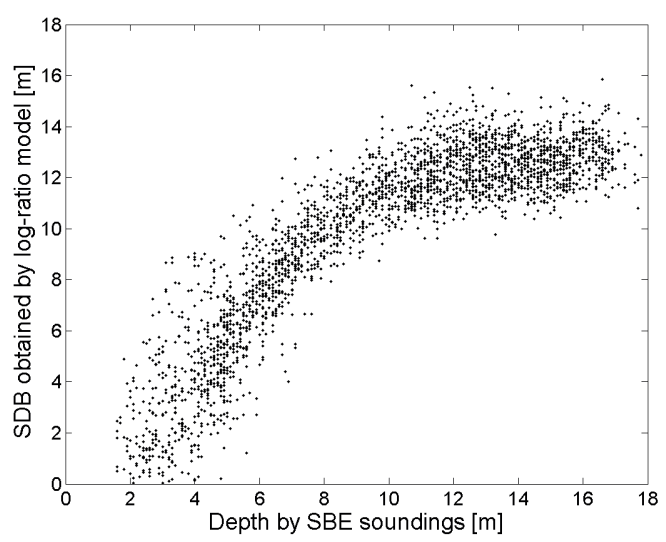

Fig. 6. Scatter plot of results obtained by optimized log-ratio model vs. depths obtained by SBE. Root mean square error was RMSE $=2.079[\mathrm{~m}]$ and Pearson correlation coefficient was $R=0.8749$. Model was calibrated on the basis of satellite acquisition made on $27^{\text {th }}$ March 2016

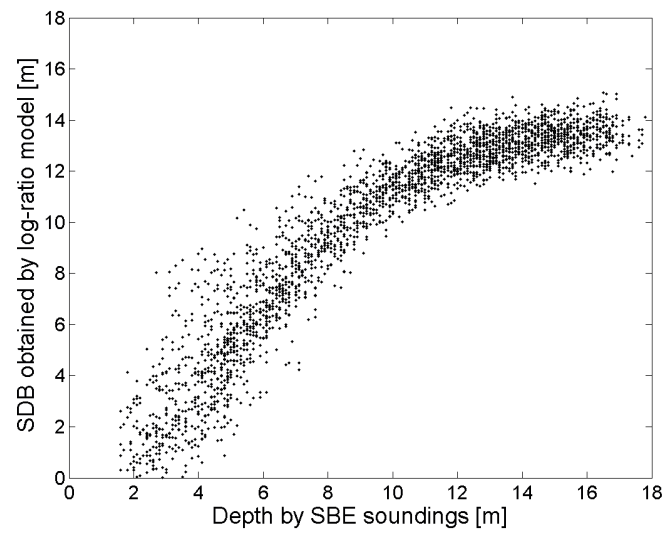

Fig. 7. Scatter plot of results obtained by optimized log-ratio model vs. depths obtained by SBE. Root mean square error was RMSE $=1.653[\mathrm{~m}]$ and Pearson correlation coefficient was $R=0.9229$. Model was calibrated on the basis of satellite acquisition made on $6^{\text {th }}$ May 2016.

The presented results indicate strong correlation between SDB and the sounding data acquired by SBE. This strong correlation can be particularly observed for mid-range bottom depths. The noise for small bottom depths is caused by optical effects of wave collapse and relatively high temporal bathymetry variation. For deeper sounding, the maximal derived bathymetry differs in different observations, however in most cases it ranges between 12 and 16 meters. Within this range of bottom depth values, the SBE data for deeper optical properties of water becomes equal to that of fully deep water.

Nevertheless, it can be seen that for each observation used to derive bathymetry, SDB depicts the characteristics of the bottom profile lines. In order to present this, selected SBE profiles were plotted in Fig. 8 together with the corresponding SDB profile. The black line represents the calibration data, while the coloured lines represent SDB derived along the selected SBE profile for different acquisition datasets. This result is consistent with previous observations, as the SDB bathymetry profile depicts the shapes of SBE bathymetry, particularly for mid-range bottom depths. The difference between SBE and SDB increases at points situated deeper than 12-14 meters.

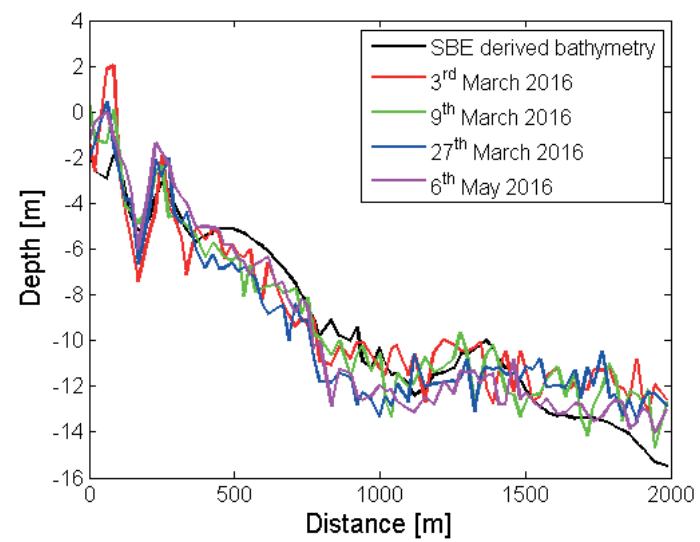

Fig. 8. Comparison of analytical model performance for different calibration datasets recorded for one selected SBE profile.

\section{ANALYTICAL MODEL CALIBRATION}

The calibration methodology for the second model was analogical, however this model consists of at least four parameters $\left(\alpha_{0}, \alpha_{1}, \alpha_{2}, \alpha_{4}\right)$ which are to be calibrated locally when using Senitnel-2 (for this purpose 3 optical bands: R, $\mathrm{G}$, and $\mathrm{B}$ were used). Thus, for the sounding point calibration dataset $M$ eq. 4 takes the form:

$$
S D B_{M(k)}=\alpha_{0}+\sum_{i=1}^{N} \alpha_{i} \ln \left[L\left(\lambda_{i}\right)_{M(k)}-L_{\infty}\left(\lambda_{i}\right)\right]
$$

where $S D B_{M(k)}$ is the satellite derived bathymetry for the $k$-th sounding point $(M(k)), k(1,2, \ldots ., K)$, and $K$ is the total number of calibration points. $L\left(\lambda_{i}\right)_{M(k)}$ is the $i$-th band reflectance corresponding to $M(k)$. Then, for $K$ calibration points, the optimal solution to the above stated optimization problem has the form of matrix equation (6):

$$
\hat{\alpha}=\left[L^{T} W L\right]^{-1} L^{T} Z
$$

where $\hat{\alpha}$ is the $N$-element column vector of optimal model parameters $\left(\alpha_{i}\right), W$ is the optional $K x K$ weight matrix, $L$ is the $K x N$ matrix, and $\mathrm{z}$ is the $K$-element column vector of sounding depths. Figures 9-12 show a series of scatter plots of calibrated SDB depths against SBE data, analogical to those shown in Figs. 4-7.

Generally, the results are consistent with those obtained in the previous case, however some significant conclusions can be derived. For instance, both models reach a similar maximum depth derivation, which ranges in about 12-16 meters. It can be also observed that higher data noise occurs for small- and maximum-depth ranges, while in mid-depth ranges the noise is relatively low. Moreover, in both models 
the SDB obtained from observation made on $6^{\text {th }}$ May 2016 returns the smallest error among all observations. That leads to the conclusion that low water turbidity and other obscuring effects have higher impact on final quality of SDB then the applied model.

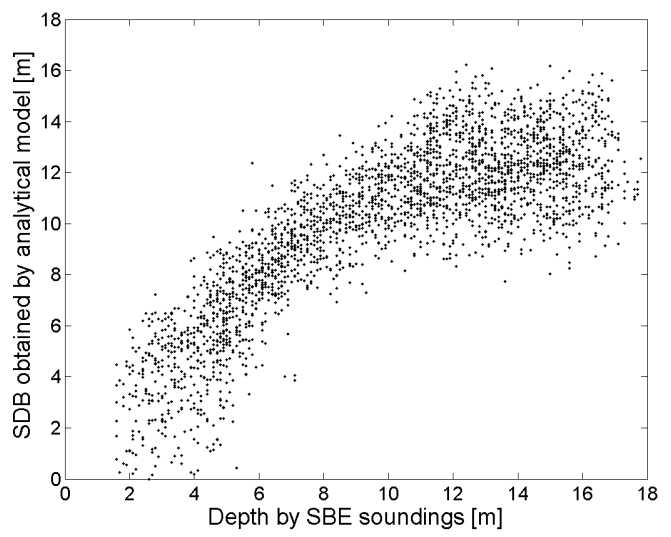

Fig. 9. Scatter plot of results obtained by optimized analytical model vs. depths obtained by SBE. Root mean square error was $R M S E=2.4186[\mathrm{~m}]$ and Pearson correlation coefficient was $R=0.8262$. Model was calibrated based on satellite acquisition made on $4^{\text {th }}$ March 2016.

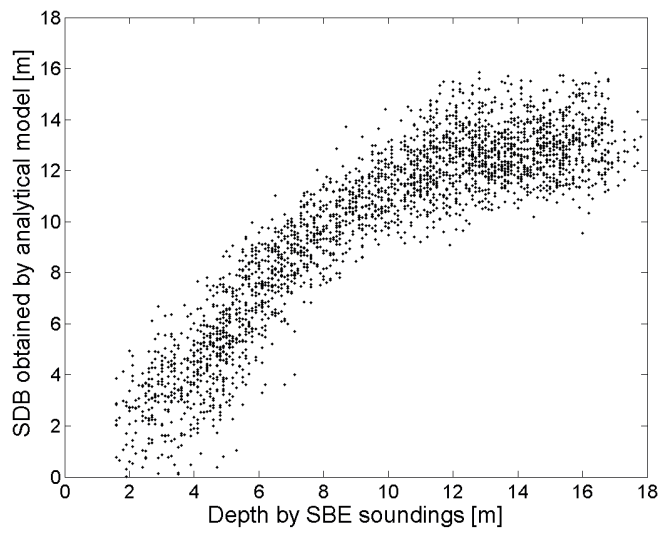

Fig. 10. Scatter plot of results obtained by optimized analytical model vs. depths obtained by SBE. Root mean square error was RMSE $=1.9694[\mathrm{~m}]$ and Pearson correlation coefficient was $R=0.885$. Model was calibrated based on satellite acquisition made on $9^{\text {th }}$ March 2016.

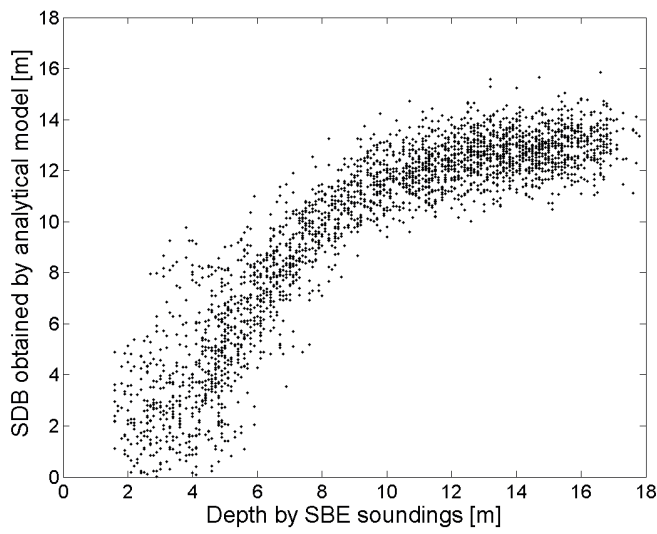

Fig. 11. Scatter plot of results obtained by optimized analytical model vs. depths obtained by SBE. Root mean square error was $R M S E=1.9727$ [m] and Pearson correlation coefficient was $R=0.8881$. 9th March 201. Model was calibrated based on satellite acquisition made on $27^{\text {th }}$ March 2016.

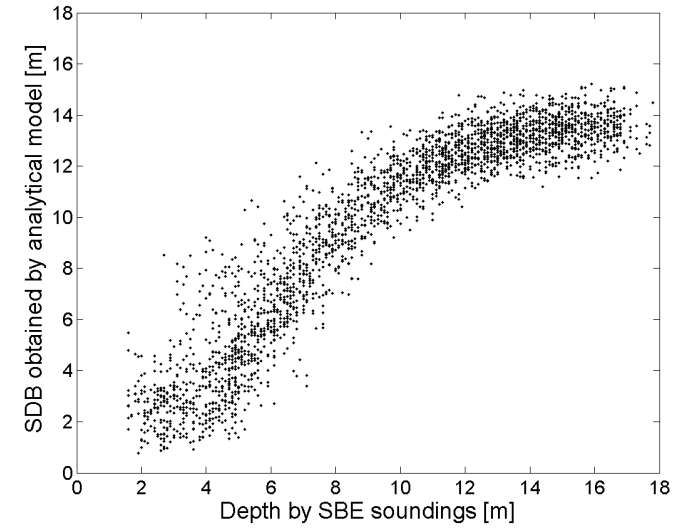

Fig. 12. Scatter plot of results obtained by optimized analytical model vs. depths obtained by SBE. Root mean square error was $R M S E=1.651[\mathrm{~m}]$ and Pearson correlation coefficient was $R=0.9231$. Model was calibrated based on satellite acquisition made on $6^{\text {th }}$ May 2016.

Detailed comparison of SDB and SBE is given in Fig. 13 for the selected SBE profile (the same as in previous case). In this case, similar conclusions related to SDB errors and maximum derivation depth can be derived. However, additional issues should be discussed. Firstly, both models can be characterized by the repeatability of the obtained SDB profiles. The shapes of the SBE bottom profiles can be observed, for all acquisitions, both in log-ratio and analytical model results. However, in both models, very shallow SDB observation can be derived as exposed over the water surface - this can be particularly seen for the observation made on $3^{\text {rd }}$ March 2016. However, there is no certain method to tell whether this information is true or false. In other words, for this particular observation, some areas placed very near to the shore could be exposed above the water surface due to low sea lever or past storm.

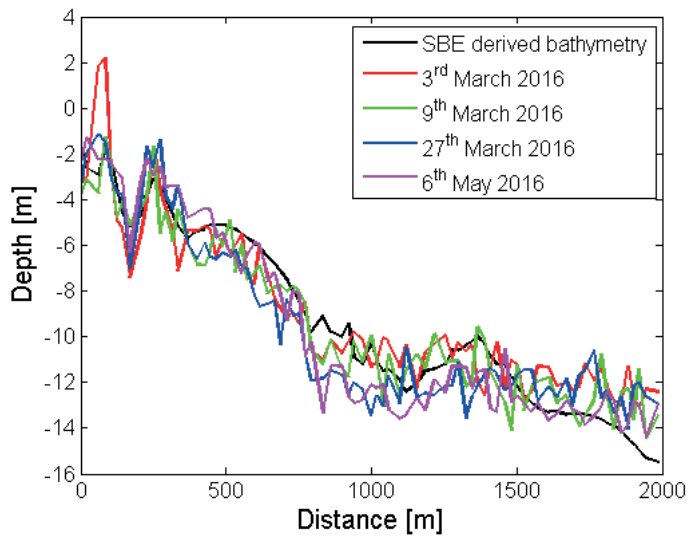

Fig. 13. Comparison of analytical model performance for different calibration datasets recorded for one selected SBE profile.

Overall comparison of model performance for different S2 observations is given in Table 1. This analysis shows that both models preserve satisfactory quality of SDB derived for depths $0-16 \mathrm{~m}$. This comparison also confirms earlier conclusions that the observation presented in the $4^{\text {th }}$ row of the table ( $6^{\text {th }}$ May 2016) is characterized by the highest quality what is confirmed by the lowest RMSE (1.653 $\mathrm{m}$ for 
the log-ratio model and $1.653 \mathrm{~m}$ for the analytical model) and highest correlation from all calibration datasets (0.9229 and 0.9231 , respectively).

Tab. 1. Comparison of model performance after calibration with different acquisition datasets

\begin{tabular}{|c|c|c|c|c|}
\hline & \multicolumn{2}{|c|}{ Log-ratio model } & \multicolumn{2}{c|}{ Analytical model } \\
\hline Acquisition date & $\mathrm{R}$ & $\mathrm{RMSE}$ & $\mathrm{R}$ & $\mathrm{RMSE}$ \\
\hline 04.03 .2016 & 0.8254 & 2.4231 & 0.8262 & 2.4186 \\
\hline 09.03 .2016 & 0.8779 & 2.0555 & 0.885 & 1.9694 \\
\hline 27.03 .2016 & 0.8749 & 2.079 & 0.8881 & 1.9727 \\
\hline 06.05 .2016 & 0.9229 & 1.653 & 0.9231 & 1.651 \\
\hline
\end{tabular}

\section{ERROR ANALYSIS}

As it was observed, the correlation between SDB and SBE bathymetry is clearly visible, particularly for smaller depths than, approximately, 16 meters. For deeper soundings, the SDB estimator becomes constant and uncorrelated, like for fully deep water. In this context, it is noteworthy that SDB models do not behave evenly for all bottom depths, and model calibration is not efficient for the depths higher than 12-16 meters. Therefore, model calibration making use of calibration points deeper than a particular threshold leads to the decrease of general model performance.

In order to analyse how bottom depth influences the error of SDB, additional analysis of the obtained results was performed. For each remote observation, the bathymetry error was plotted as the function of depth. The results of this analysis are given in Fig. 14-17. In each figure, the upper plot represents the SDB error as the function of depth, the middle graph represents the number of calibration points for each water depth bin (it is a depth histogram of the calibration dataset). The lower plot shows changes of the introduced SDB quality coefficient $\left(S D B_{Q \text { coef }}\right)$ that describes the quality of the retrieved SBD. This coefficient is described by the following formula:

$$
S D B_{Q c o e f}=\frac{\ln \left(1+R\left(\lambda_{i}\right)\right)}{\ln \left(1+R\left(\lambda_{j}\right)\right)}-\left(\ln \left[\frac{1+R_{\infty}\left(\lambda_{i}\right)}{1+R_{\infty}\left(\lambda_{j}\right)}\right]-2 \sigma\right)
$$

where $\sigma$ is the standard deviation of the selected field with fully deep-water log ratios reflectance for wavelengths $\lambda_{i}$ and $\lambda_{j} . S D B_{\text {Qcoef }}$ enables to determine a threshold to which bathymetry derived by the proposed models can be retrieved with certain quality. Note that this value depends only on the remote observation data, therefore neither bathymetric nor other auxiliary information is necessary to compute it. Consequently, it can be used operationally. The red line in the lower plots (Fig. 14-17) represents the threshold equal to 0 . As it can be observed, as $S D B_{\text {Qcoef }}$ reaches this threshold, the $\mathrm{SDB}$ error is clearly increasing.

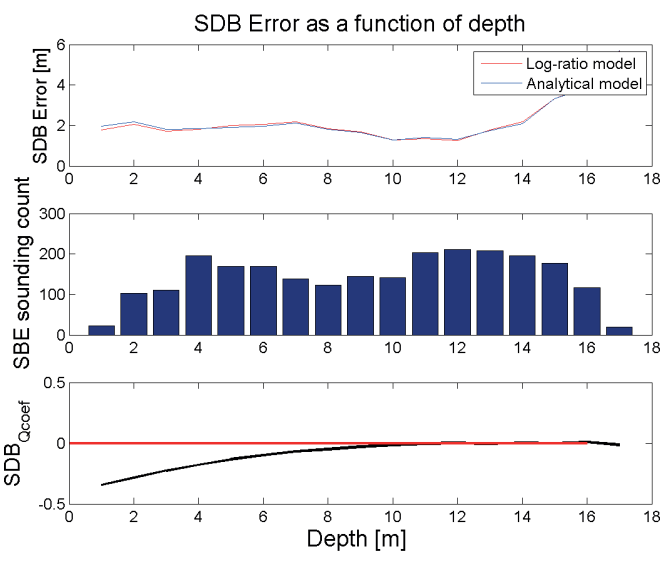

Fig. 14. Error distribution as function of depth (upper picture), SBE sounding count (middle), and SDB quality indicator plot (lower) for $4^{\text {th }}$ March 2016. The red line in the lower plot represents threshold equal to 0.

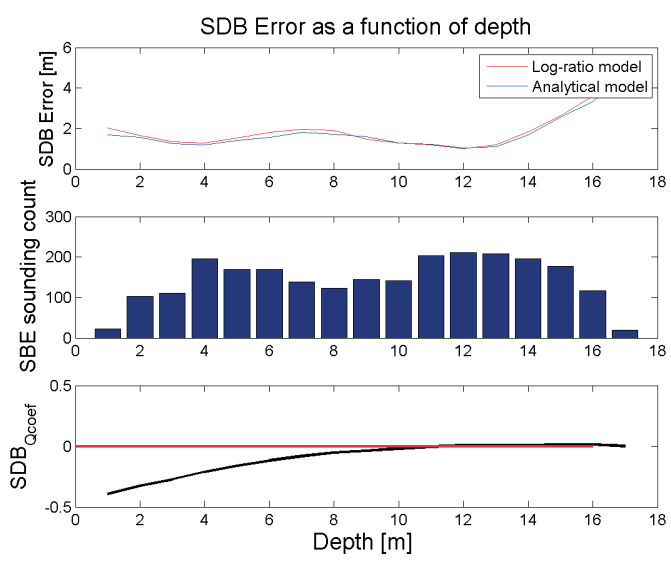

Fig. 15. Error distribution as function of depth (upper picture), SBE sounding count (middle), and SDB quality indicator $\left(S D B_{Q \text { coef }}\right)$ plot (lower) for $9^{\text {th }}$ March 2016

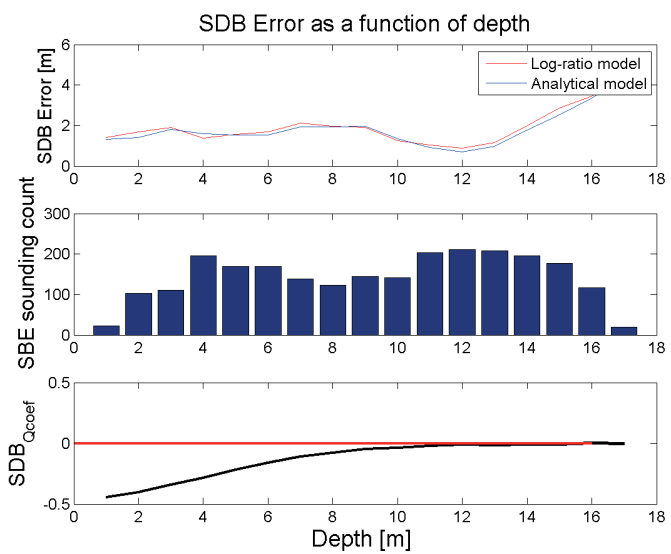

Fig. 16. Error distribution as function of depth (upper picture), SBE sounding count (middle), and SDB quality indicator $\left(S D B_{Q \operatorname{coe}}\right)$ plot (lower) for $27^{\text {th }}$ March 2016 


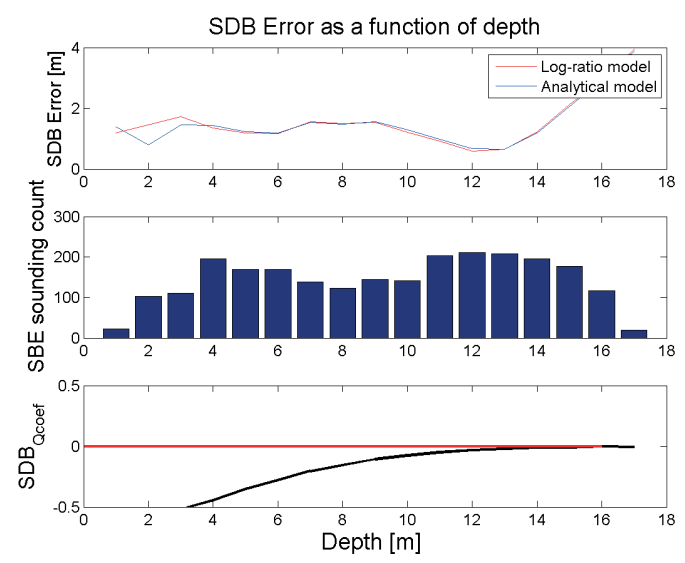

Fig. 17. Error distribution as function of depth (upper picture), SBE sounding count (middle), and SDB quality coefficient $\left(S D B_{Q c o e f}\right.$ ) indicator plot (lower) for $6^{\text {th }}$ May 2016

In order to show benefits of using the $S D B_{\text {Qcoef }}$ threshold, each model was calibrated with only those observations which met the aforementioned quality criterion, namely where $S D B_{\text {Qcoef }}<0$. In this way, only those observations were used for model calibration which were indicated as valid by the quality indicator. The results of model calibration presented in Tab. 2 reveal significant improvement of model performance. Namely, for each observation dataset, the RMSE values are significantly lower. For the log-ratio model the RMSE was reduced from the range of 1.653-2.4231[m] to 1.0822-1.4319 [m], while for the analytical model the final RMSE values ranged within 1.0681-1456[m], compared to the initial range of 1.651-2.4186 [m]. Analogically, the increase of the correlation coefficient was observed from 0.8254-0.9229 to $0.8736-0.9346$ for the log-ratio model and from 0.8262 0.9231 to 0.869-0.9345 for the analytical model.

Tab. 2. Comparison of model performance after calibration with different SDB acquisition datasets and maximal depth determined by the quality indicator (SDBQcoef).

\begin{tabular}{|c|c|c|c|c|}
\hline & \multicolumn{2}{|c|}{ Log-ratio model } & \multicolumn{2}{c|}{ Analytical model } \\
\hline Acquisition date & $R$ & RMSE & $R$ & RMSE \\
\hline 04.03 .2016 & 0.8736 & 1.4319 & 0.869 & 1.456 \\
\hline 09.03 .2016 & 0.9065 & 1.0822 & 0.9091 & 1.0681 \\
\hline 27.03 .2016 & 0.9083 & 1.3678 & 0.9115 & 1.3447 \\
\hline 06.05 .2016 & 0.9346 & 1.3632 & 0.9345 & 1.3643 \\
\hline
\end{tabular}

\section{VERIFICATION OF RESULTS}

In order to perform visual inspection of bottom maps generated by the proposed SDB models and visually verify the quality of these models, maps representing the bathymetry derived from remote observations were generated. Figures 18-21 represent SDB images obtained with the use of the logratio model and the analytical model. The data is presented as colour coded depths ranging from 0 to 18 meters. The analytical model was calibrated with the use of $S D B_{\text {Qcoef }}$ thresholding technique, described in the previous section. The areas above water surface, in SDB and coast, are marked as white pixels.
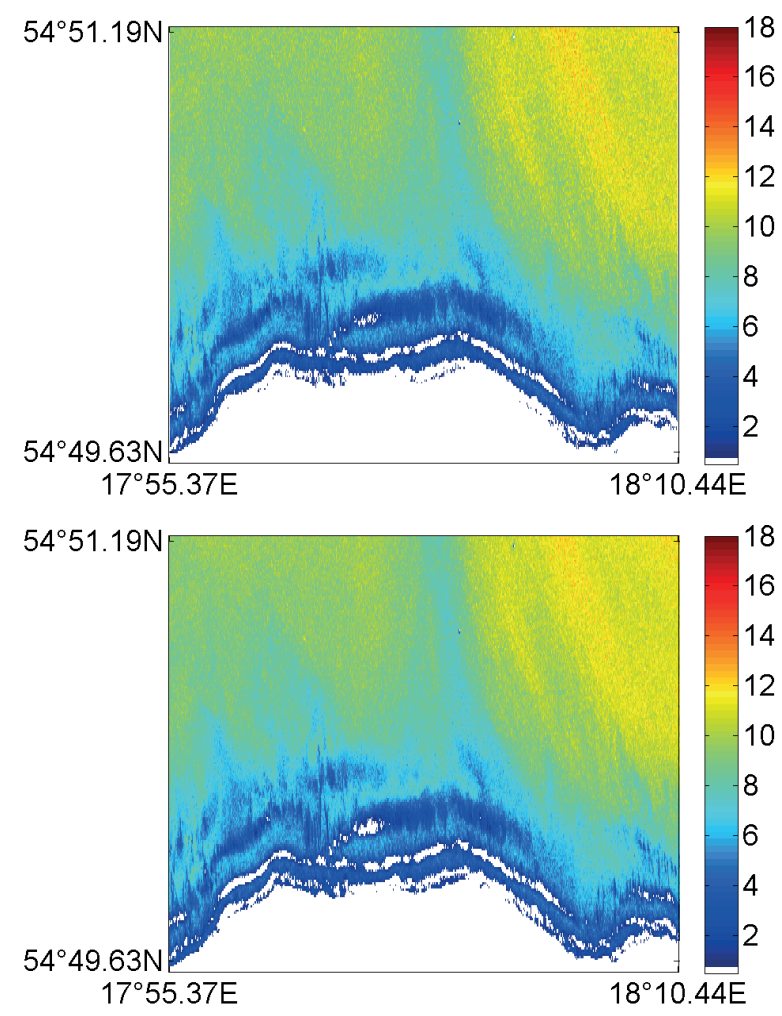

Fig. 18. Comparison of colour coded depth maps obtained by the optimized log-ratio SDB model (upper figure) and the analytical SDB model (lower figure) for $3^{\text {rd }}$ March 2016.
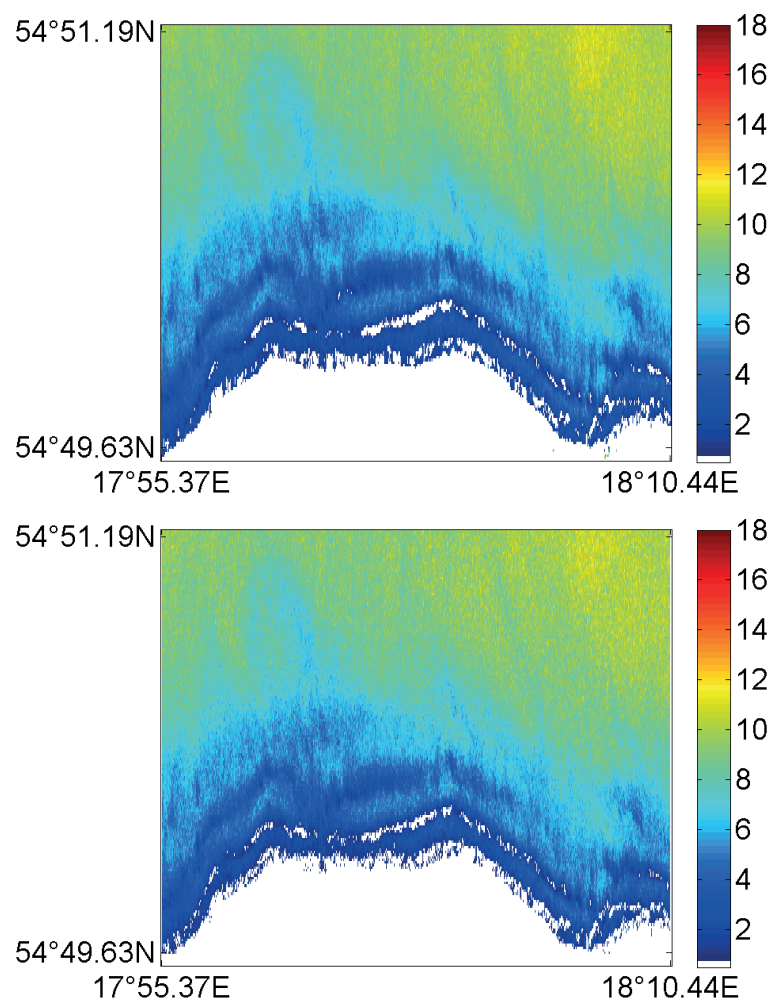

Fig. 19. Comparison of colour coded depth maps obtained by the optimized log-ratio SDB model (upper figure) and the analytical SDB model (lower figure) for 9th March 2016. 

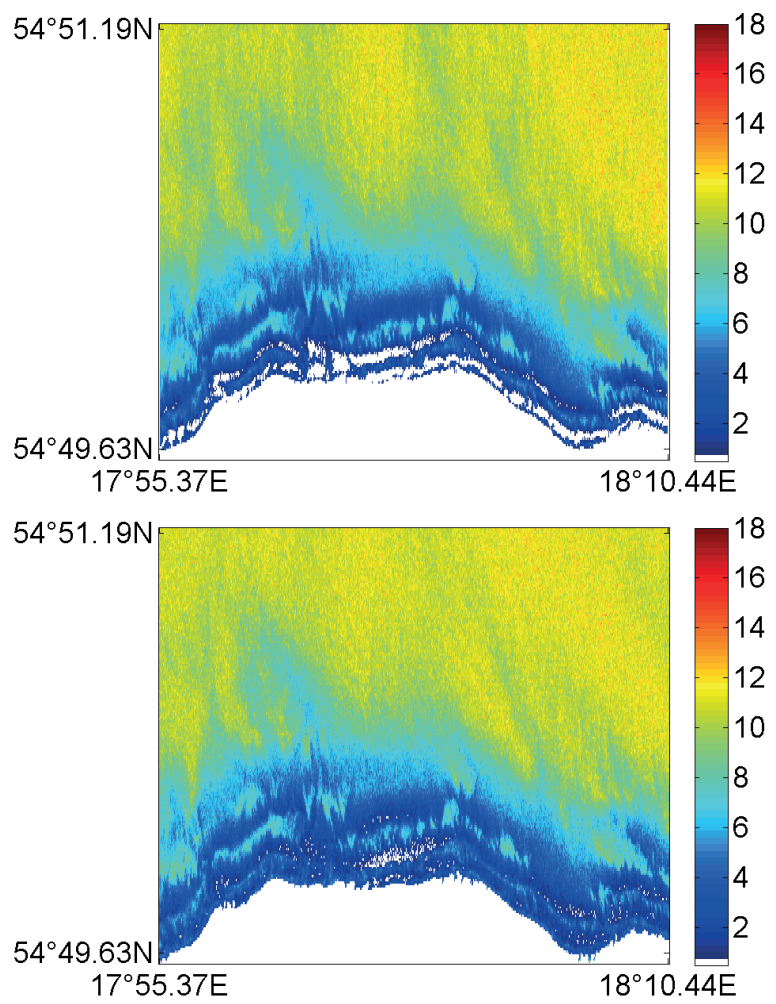

Fig. 20. Comparison of colour coded depth maps obtained by the optimized log-ratio SDB model (upper figure) and the analytical SDB model (lower figure) for $27^{\text {th }}$ March 2016. White pixels represent depths derived as situated above the sea surface.
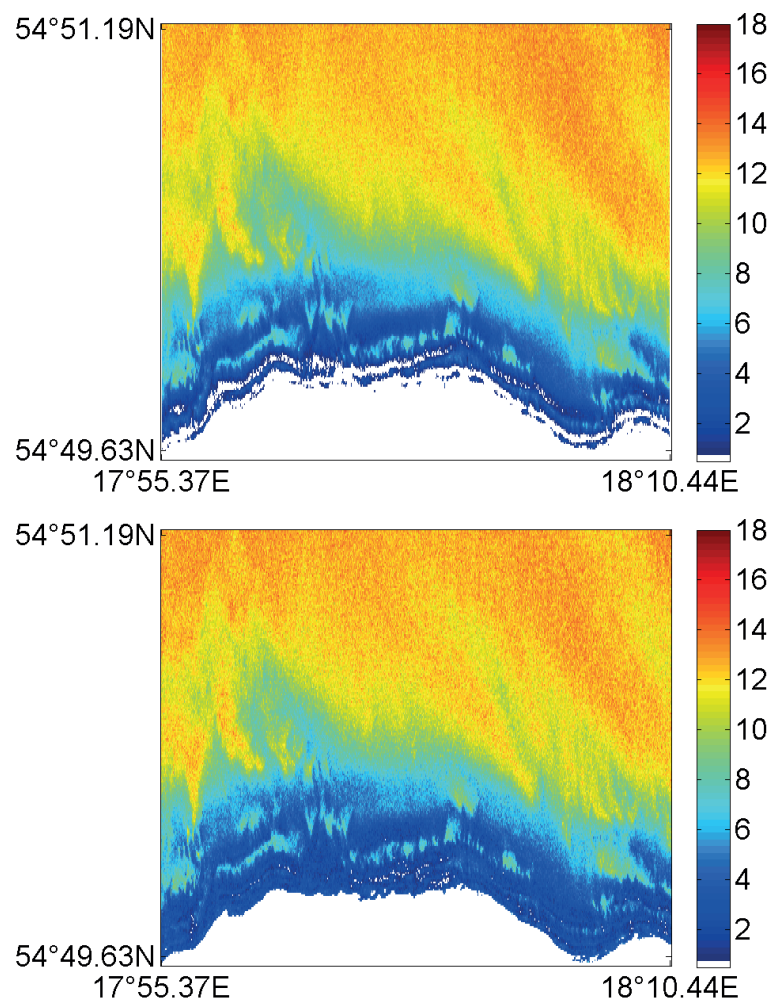

Fig. 21. Comparison of colour coded depth maps obtained by the optimized log-ratio SDB model (upper figure) and the analytical SDB model (lower figure) for $6^{h}$ May 2016. White pixels represent depths derived as situated above the sea surface.
It can be observed that the corresponding depth maps retrieved from both models are relatively similar to each other, and both techniques enable to obtain similar results in the context of visual analysis. Visible noise levels observed in the scatter plots presented in previous sections (Fig. 4-11) are also noticeable in these maps. Visual inspection of the results also reveals the fact that the examined methods behave relatively poorly for depths exceeding the maximum derivation depth determined by the $S D B_{\text {Ocoef }}$ factor. However, for shallow and non-turbid waters, even single 3D shapes of underwater bathymetry are easy to retrieve for human eye.

Visual inspection enables to notice only minor differences between the observations acquired at the same time, and both methods can be successfully used in relatively turbid and difficult conditions.

\section{CONCLUSIONS}

The paper compares two fundamental methods for bathymetry retrieval from S2 multispectral satellite observations. The results of model performance were obtained using the data acquired for the $12-\mathrm{km}$ long South Baltic coastline. The calibration points acquired from SBE surveys were delivered by the National Maritime Administration.

A detailed analysis of the obtained results shows that both methods can be successfully applied for the South Baltic region. However, some limitations and factors causing obstruction of the results can be observed. It is water turbidity which is most important in this case, therefore the bathymetry can be derived to the depth approximately equal to 12-18 meters. What is also important is the fact that the maximum depth that can be derived from satellite observation varies in time and space and is difficult to be assumed a priori. To overcome this drawback, a novel indicator of determining maximal SDB depth was proposed in the paper. This SDB quality indicator is derived only on the basis of the remotely registered data and can therefore be applied operationally.

During the research, a detailed analysis of errors obtained for different depth ranges was also performed. The obtained results indicate that the error of model calibration, expressed in meters (RMSE), equals up to $10-20 \%$ of the real depth and is, generally, case dependent. This value is worse than the results obtained by other authors [1][3-16]. However, there are at least two reasons for this. The first is water turbidity, as the effects of light attenuation and its spatial variety are much more obscuring in the Baltic Sea than in other locations such as presented in [1][3-16], where the testing datasets were related to optically clear waters. Another issue is that bathymetry surveys for this research were not collected at the same time. This is because of the fact that the Polish Maritime office performs SBE surveys periodically and, consequently, exact time co-incidence between remote observation and calibration data acquisitions was unavailable for our test site. Nevertheless, it was shown that the proposed methods, combined with the SDB quality indicator, are not only selfadaptive but can also be used operationally, for instance to 
deliver a cost-effective alternative for large scale bathymetry observations. The novelty of the research presented in the paper also relies on the fact that it bases on newly deployed ESA Sentinel-2 observations obtained for relatively difficult turbid Baltic Sea waters.

\section{ACKNOWLEDGEMENTS}

The author wishes to thank the Maritime Office in Gdynia, Poland, for providing sounding bathymetry maps used in the research presented in the paper.

\section{REFERENCES}

1. D. R. Lyzenga, N. R. Malinas, and F. J. Tanis, "Multispectral bathymetry using a simple physically based algorithm," IEEE Trans. Geosci. Remote Sens., vol. 44, no. 8, pp. 22512259, Aug. 2006.

2. A. Chybicki et al., "GIS for remote sensing, analysis and visualisation of marine pollution and other marine ecosystem components," 2008 1st International Conference on Information Technology, Gdansk, 2008, pp. 1-4. doi: 10.1109/INFTECH.2008.4621628

3. Stumpf, R.P., Holderied, K., Sinclair, M., 2003. Determination of water depth with high-resolution satellite imagery over variable bottom types. Limonology Oceanogr. 48, 547-556. doi:10.4319/lo.2003.48.1_part_2.0547

4. R. Lyzenga, D., 1981. Remote sensing of bottom reflectance and water attenuation parameters in shallow water using aircraft and Landsat data. Int. J. Remote Sens. 2, 71-82.

5. R. Lyzenga, D., 1978. Passive remote sensing technique for mapping water depth. Appl. Opt. 17, 379-383.

6. Philpot, W.D., 1989. Bathymetric mapping with passive multispectral imagery. Appl. Opt. 28, 1569-1578. doi:10.1364/AO.28.001569

7. J. C. Sandidge, and R. J. Holyer, "Coastal bathymetry from hyperspectral observations of water radiance," Remote Sens. Environ., vol. 65, no. 3, pp. 341-352, Sep. 1998.

8. S. M. Adler-Golden, P. K. Acharya, A. Berk, M. W. Matthew, and D. Gorodetzky, "Remote bathymetry of the littoral zone from AVIRIS, LASH, and QuickBird imagery," IEEE Trans. Geoscis. Remote Sens., vol. 43, no. 2, pp. 337-347, Feb. 2005.

9. Sheng Ma, Zui Tao, Xiaofeng Yang, Member, IEEE, Yang $\mathrm{Yu}$, Xuan Zhou, and Ziwei Li, "Bathymetry Retrieval from Hyperspectral Remote Sensing Data in Optical-Shallow Water", Ieee Transactions on Geoscience and Remote Sensing, Vol. 52, No. 2, February 2014
10. H. Holden and E. LeDrew, "Measuring and modeling water column effects on hyperspectral reflectance in a coral reef environment," Remote Sens. Environ., vol. 81, nos. 2-3, pp. 300-308, Aug. 2002.

11. Haibin Su, Hongxing Liu, William D. Heyman, "Automated Derivation of Bathymetric Information from MultiSpectral Satellite Imagery Using a Non-Linear Inversion Model", Marine Geodesy, vol. 31, 2008.

12. E. P. Green, P. J. Mumby, A. J. Edwards, and C. D. Clark, "Remote sensing handbook for tropical coastal management," in Coastal Management Sourcebooks 3, A. J. Edward, Ed. Paris, France: UNESCO, 2000.

13. Poliyapram V., Venkatesh R., Shinji M., 2016, Satellitederived bathymetry using adaptive geographically weighted Regression model, Marine Geodesy, vol. 39:6, 458-478.

14. Jensen, J. R. 2007. Remote sensing of the environment: An earth resource perspective, 2nd ed. Upper Saddle River, NJ: Prentice Hall

15. Mishra, D., S. Narumalani, D. Rundqulst, and M. Lawson. 2006. Benthic habitat mapping in tropical marine environments using QuickBird multispectral data. Photogrammetric Engineering \& Remote Sensing 72:1037-1048.

16. Lyzenga, D. R., N. P. Malinas, and F. J. Tanis. 2006. Multispectral bathymetry using a simple physically based algorithm. Geoscience and Remote Sensing, IEEE Transactions on 44:2251-2259.

17. Green, E. P., P. J. Mumby, A. J. Edwards, and C. D. Clark. 2000. Remote sensing handbook for tropical coastal management. Paris: A. J. Edwards, UNESCO

18. Drusch, M. \& 14 co-authors (2012). Sentinel-2: ESA's optical high-resolution mission for GMES operational services, Rem. Sens. Env. (accepted).

19. Sentinels Scientific Data Hub, Technical Guide, European Space Agency, source: https://scihub.copernicus.eu/ userguide/ (accessed on 22/12/2016)

20. Hellenic National Sentinel Data Mirror Site. Operated by the National Observatory of Athens. Source: https:// sentinels.space.noa.gr/ (accessed on 22/12/2016)

21. National French Copernicus Site, Centre National D'Etudes Spatiales, Source: https://copernicus.cnes.fr/en/groundsegment-1 (accessed on 22/12/2016) 
22. Sentinel Application Platform (SNAP), available at Science Toolbox Exploitation Platform (STEP), European Space Agency, source: http:/step.esa.int/main/toolboxes/snap/ (accessed on 22/12/2016)

23. Sen 2 cor - Sentinel-2 Level 2 A product generation and formatting, available at Science Toolbox Exploitation Platform (STEP), European Space Agency, source: http:// step.esa.int/main/third-party-plugins-2/sen2cor/ (accessed on $22 / 12 / 2016$ )

\section{CONTACT WITH THE AUTHOR}

\section{Andrzej Chybicki}

e-mail: andrzej.chybicki@eti.pg.gda.pl Gdansk University of Technology Narutowicza 1/2, 80-233 Gdansk

Poland 